\title{
A Secondary Fingerprint Enhancement and Minutiae Extraction
}

\author{
Raju Rajkumar ${ }^{1}$, K Hemachandran ${ }^{2}$ \\ Department of Computer Science Assam University, Silchar, India \\ ${ }^{1}$ rajurajkumar.phd@gmail.com , ${ }^{2}$ khchandran@rediffmail.com
}

\begin{abstract}
In this paper, we introduce a new fingerprint enhancement algorithm which used FFT and Gaussian filter. FFT is applied on a block, of size $32 \times 32$ pixel and Gaussian filter is applied in each interconnection of each block. This make ridge smoothness and also reduce the "hairy" structure in the ridge when it is thinning. The experimental result shows that the implementation of FFT and Gaussian filter in the preprocessing stage make less effort in the postprocessing stage and hence the total error rate of the proposed algorithm is very low.
\end{abstract}

\section{KEYWORDS}

Primary enhancement, secondary enhancement, real minutiae, spurious minutiae.

\section{INTRODUCTION}

Different biometric methods like palm print, finger print, iris, signature, retina, and face are widely being used in personnel identification systems and the fingerprint based identification is used even in ancient times. A fingerprint is made of a series of ridges and furrows on the surface of the finger. The uniqueness of a fingerprint can be determined by the pattern of ridges and furrows as well as the minutiae points (Lee and Gaensslen, 1991). Minutiae points are local ridge characteristics that occur at either a ridge bifurcation or a ridge ending as shown in Figure 1.

Several factors like the presence of scars, variations of the pressure between the finger and the image acquisition sensor, the environmental conditions during the acquisition process, worn artifacts and so forth, can dramatically affect the quality of the acquired fingerprint image. Since minutiae depend on the fine details of the ridge pattern, their extraction can become notoriously difficult if the noise generated by the factors described above is not substantially reduced. This brings the necessary need of the fingerprint 
Signal \& Image Processing : An International Journal (SIPIJ) Vol.3, No.2, April 2012

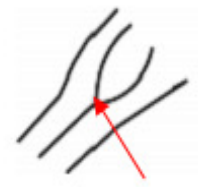

a) Ridge bifurcation

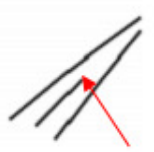

b) Ridge ending

Figure1 Two types of fingerprint minutiae

enhancement to reduce unwanted noise. Fingerprint enhancement is different from general image enhancement method (Rajkumar and Hemachandran, 2011). Lots of fingerprint enhancement algorithm has been proposed in the literature, with a aim to improve the minutiae structure. Gorman and Nickerson (1989) have proposed different filters for the fingerprint image enhancement and the $\mathrm{k} \mathrm{X} \mathrm{k} \mathrm{mask} \mathrm{coefficients} \mathrm{are} \mathrm{generated,} \mathrm{based} \mathrm{on} \mathrm{the} \mathrm{local} \mathrm{ridge} \mathrm{orientation.}$ The four model parameters, derived from ridge width $\left(\mathrm{W}_{\max }, \mathrm{W}_{\min }\right)$, valley width $\left(\mathrm{W}^{-}{ }_{\max }, \mathrm{W}^{-}{ }_{\min }\right)$ and the minimum radius of curvature are used to describe a fingerprint. It is assumed that the $\mathrm{W}_{\text {max }}+\mathrm{W}_{\text {min }}=\mathrm{W}^{-}{ }_{\text {max }}+\mathrm{W}^{-}{ }_{\text {min }}$. The mask is convoled with the input image. The enhanced image is binarized and post processed for fingerprint identification. Mehtre (1993) described a directional image of $16 \times 16$ pixel block and computed local gray level intensity variance along eight directions. The desired least direction is the direction which has least variance. The

foreground and background of the fingerprint image is segmented by using local adaptive threshold and a Crossing Number $(\mathrm{CN})$ is used for minutiae extraction. Sherlock, Monro and Millard (1994) described a directional Fourier filter for the fingerprint image which used the information from the entire image, instead of a small neighborhood of the filtered point. The enhancement has two stages, like filtering stage and threshold stage. The filtering stage is for producing a directional smooth image by removing the noise from the input image and threshold stage is for converting the gray scale enhanced image in to binary image. Hong, Jain, Pankanti and Bolle (1996) have taken advantage of the Gabor filter( Gabor 1946) frequency selective and orientation selective properties, to introduce a fiingerprint enhancement algorithm, with a set of band pass filters, to remove the noise and preserve the true ridge and furrow structures. Hong, Wan, and Jain (1998) developed a fast enhancement algorithm, suitable for online fingerprint verification system. The algorithm finds the input fingerprint image into three regions as a well defined region, a recoverable corrupted region and an unrecoverable corrupted region and the Gabor filter is applied, only on the first two regions for enhancement and simply discarded the unrecoverable corrupted region.. Hong, Wan and Jain (1998) have used the traditional Gabor filter, assuming the ridge orientation as a sinusoidal plan wave, for enhancement. Yang, Liu, Jiang and Fan (2003) have modified the traditional Gabor filter with the parameter selection process, independent of the input fingerprint image. Xiao and Raafat (1991) considered the skeleton image as an input image and proposed an algorithm which identifies spurious minutiae and eliminates using the structural definition of the minutiae. The spurious minutiae are identified by using ridge width and ridge attributes like ridge direction, ridge length and minutiae direction. Hung (1993) used Baja's algorithm to obtained a skeleton fingerpint image. The false minutiae are eliminated by evaluating the statistical parameters within an M X M matrix. Chen and Kuo (1992) adopt a three-step false minutiae identification and elimination process like: (i) ridge breaks are repaired, using the ridge directions close to the minutiae; (ii) minutiae associated with short ridges are dropped; (iii) crowded minutiae in a noisy region are dropped. 
Signal \& Image Processing : An International Journal (SIPIJ) Vol.3, No.2, April 2012

\section{PROPOSED ALGORITHM}

Automatic Fingerprint Recognition System requires several stages of processing to match an unknown fingerprint image for identification. Each and every processing stage in the system has its own contribution to make an accurate match or a miss match. Fingerprint identification or verification process is highly dependent on the enhancement stage and feature extraction stage. If the undesired noise, which contributes to the false minutiae, is removed in the enhancement stage and only genuine minutiae are extracted during the feature extraction stage than the matching accuracy, for personal identification, is better, but in practice it is not possible due to the presence of noise intern false minutiae even after enhancement. In order to make more effective identification/verification system, a multi stage processing system is proposed. The algorithm is divided into three parts like preprocessing stage, post processing stage and matching stage as given in the figure 2. The input fingerprint image is processed for skeleton image by the preprocessing stage and subsequently processed by post processing stage for minutiae, used in matching stage. The preprocessing and postprocessing stages are explained in the following section.

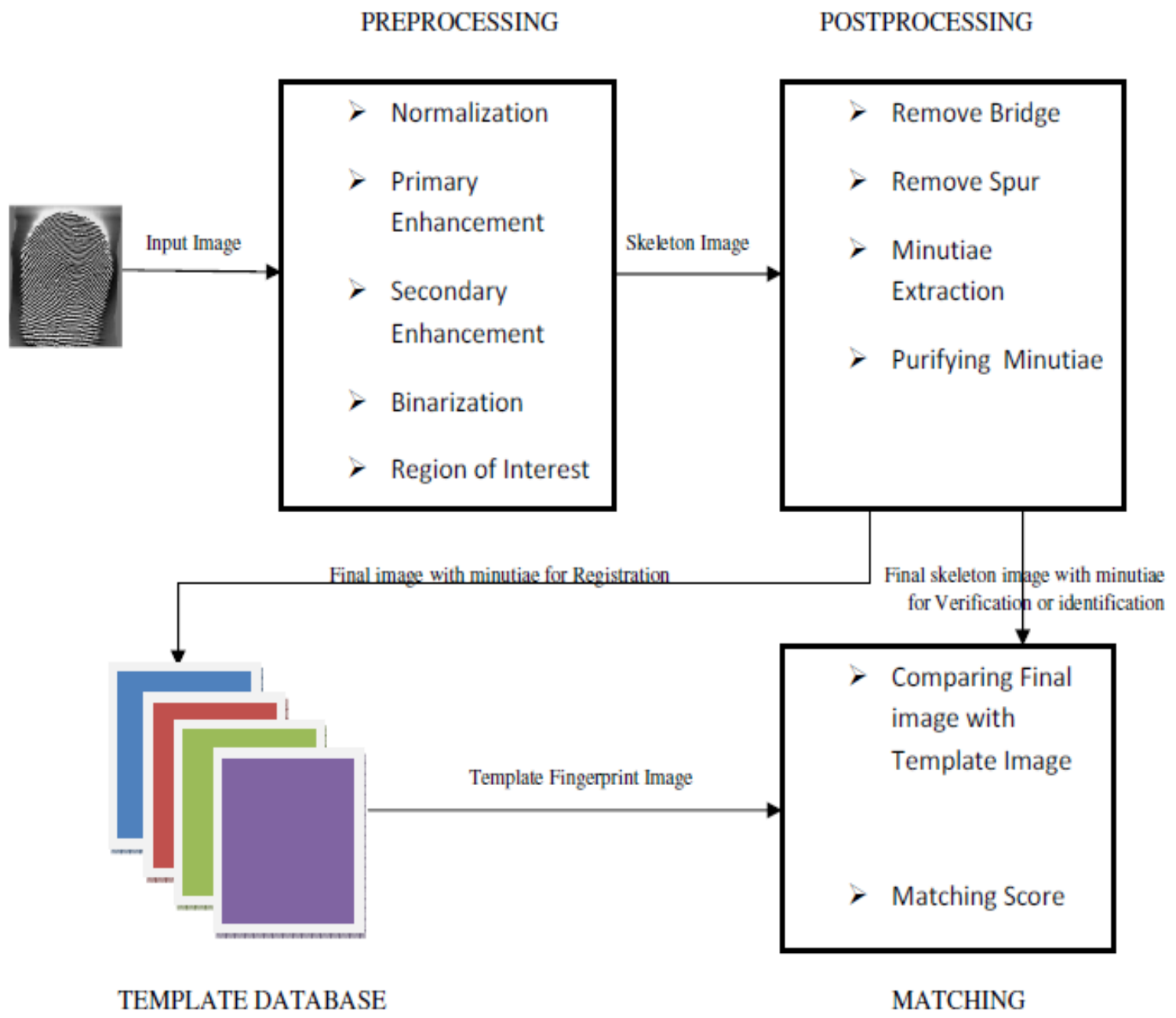

Figure 2. Different stage of proposed algorithm 
Signal \& Image Processing : An International Journal (SIPIJ) Vol.3, No.2, April 2012

\subsection{Normalization}

The Normalization is performed through histogram equalization to increase the local contrast in an image by intensity distribution on the histogram. Through histogram equalization the lower local contrast gain a higher contrast without affecting a global contrast.

\subsection{Primary Enhancement}

The primary enhancement is performed on the input fingerprint image to remove the noise from the ridge and furrow structure of the noisy fingerprint image. Some of the primary enhancement methods available in the literature are Gabor filter, Short term Fourier transformation (STFT), Fast Fourier transformation (FFT) which are based on directional filters.

The primary enhancement using the Fourier transformation technique is achieved by dividing the image into small processing blocks of size $32 \times 32$ pixels and the Fourier transformation is performed on each block according to the following equation:

$$
\begin{gathered}
F(u, v)=\sum_{x=0}^{M-1} \sum_{y=0}^{N-1} f\left(x_{z} y\right) * \exp \left\{-j 2 \pi *\left(\frac{u x}{M}+\frac{v y}{N}\right)\right\} \\
\text { for } \mathrm{u}=0,1,2, \ldots, 31 \text { and } \mathrm{v}=0,1,2, \ldots, 31
\end{gathered}
$$

The sub-divided block are enhanced by their dominant frequencies by multiplying the FFT of the block by its magnitude a set of times and the magnitude of the FFT is obtained as $|\mathrm{F}(\mathrm{u}, \mathrm{v})|$.

The enhanced block is obtained according to

$$
g(x, y)=F^{-1}\left\{F(u, v) *|F(u, v)|^{K}\right\}
$$

Where $\mathrm{k}$ is an experimentally determined constant, as 0.45 . While having a higher " $\mathrm{k}$ " value improves the appearance of the ridges, by filling up small holes in ridges and having a too high " $\mathrm{k}$ " value can result in false joining of ridges. Thus a termination might become a bifurcation and the inverse transform is given as:

$$
f(x, y)=\frac{1}{M N} \sum_{x=0}^{M-1} \sum_{y=0}^{N-1} F(u, v) * \exp \left\{j 2 \pi *\left(\frac{u x}{M}+\frac{v y}{N}\right)\right\}
$$

$$
\text { for } \mathrm{x}=0,1,2, \ldots, 31 \text { and } \mathrm{y}=0,1,2, \ldots, 31 \text {. }
$$

The enhanced image due to Fourier Transformation has the improved ridge structure by connecting some falsely broken points on the ridge and removing some spurious connection between the ridges. However, the alignment of the ridges in the sub-blocks of the images may lead to some spurious connection which can be remove in the secondary enhancement stage. 
Signal \& Image Processing : An International Journal (SIPIJ) Vol.3, No.2, April 2012

\subsection{Secondary enhancement}

A Secondary enhancement is perform using a two dimensional Gaussian filter given by

$$
f(x, y)=e^{-\frac{x^{2}+y^{2}}{2 \sigma^{2}}}
$$

Where $\sigma$ is the standard deviation. A large value of $\sigma$ produces a flatter curve and a small value leads to a "pointier" curve. In this study, the value of $\sigma$ is 1.5 .

The Secondary enhancement is required to reduce the "hairy" (spikes) structures which lead to spurious ridge bifurcations and endings. More over the mismatch or alignment of the blocks in FFT are removed by Gaussian filter.

\subsection{Binarization}

In the literature, features are directly extract from gray scale image ( Maio and Maltoni, 1997; Zhao, Zhang, David and Luo, 2009; Arpit and Namboodiri, 2011) and some are converting gray scale to binary image as one of the step for distinguish ridge flow from background in fingerprint preprocessing algorithm. Some of the existing methods for fingerprint image to convert gray scale to binary image are back propagation algorithm, local adaptive binarization method, binarization using convex threshold and double threshold binarization method etc.

A locally adaptive binarization method is used for this algorithm. Such a named method comes from the mechanism of transforming a pixel value to 1 when the value is larger than the mean intensity value of the current block (16x16) to which the pixel belongs.

\subsection{Ridge direction}

The ridge direction is to describe the ridge and furrow flow structure which are tightly bound together to represent a fingerprint images. The ridge direction stage is helpful for segmenting foreground and background in Region of Interest stage.

The algorithm for the estimation of the direction of each block of the fingerprint image with $\mathrm{W} x$ $\mathrm{W}$ size (where $\mathrm{W}$ is 16 pixels by default) is given below:

a) The gradient values, along $\mathrm{x}$-direction $\mathrm{g}_{\mathrm{x}}$ and $\mathrm{y}$-direction $\mathrm{g}_{\mathrm{y}}$, for each pixel of the block, are calculated using two Sobel filters.

b) For all the pixels in each block, the least square approximation of the block direction is obtained using the formula:

$\operatorname{tg} 2 \beta=2 \sum \sum\left(g_{x} * g_{y}\right) / \sum \sum\left(g_{x} 2-g_{y}{ }^{2}\right)$

The formula is understood by considering the gradient values along $\mathrm{x}$ and $\mathrm{y}$ directions as cosine and sine values respectively. Accordingly, the tangent value of the block direction is expressed as:

$$
\operatorname{tg} 2 \theta=2 \sin \theta \cos \theta /\left(\cos ^{2} \theta-\sin ^{2} \theta\right)
$$


Signal \& Image Processing : An International Journal (SIPIJ) Vol.3, No.2, April 2012

c) After the estimation of each block direction, the blocks without significant information on the ridges and furrows are discarded, based on a threshold for a certain level E:

$$
\mathrm{E}=\left\{2 \sum \sum\left(\mathrm{g}_{\mathrm{x}} * \mathrm{~g}_{\mathrm{y}}\right)+\sum \sum\left(\mathrm{g}_{\mathrm{x}}{ }^{2}-\mathrm{g}_{\mathrm{y}}{ }^{2}\right)\right\} / \mathrm{W} * \mathrm{~W} * \sum \sum\left(\mathrm{g}_{\mathrm{x}}{ }^{2}+\mathrm{g}_{\mathrm{y}}{ }^{2}\right)
$$

\subsection{Region of Interest}

The Region Of Interest is for separating the fingerprint foreground image from the background image. This segmentation process is required before thinning process to represent the ridge structure correctly, otherwise some background noise also will contribute to the ridge (short distance ridge) and it will be present when minutiae are being extracted from the thinning images.

The morphological operations OPEN and CLOSE are used for the determination of the Region Of Interest (ROI). The tightly bounded region is obtained by removing the leftmost, rightmost, uppermost and bottommost blocks through the algorithm using the morphological operations.

\subsection{Thinning}

Ridge thinning is to eliminate the redundant pixels of the ridges till the ridge is just one pixel wide. No further removal of the pixels should be possible after completion of thinning process. The proposed algorithm uses the built-in Morphological thinning function available in MATLAB image processing tool box. The objective of a good thinning algorithm is:

a) To obtain a thinned fingerprint image with a single pixel width and no discontinuities.

b) To eliminate the noise and singular pixels.

A typical skeleton image fingerprint pattern after thinning process without secondary enhancement and with secondary enhancement are shown in figure 3(A) and 3(B) respectively.

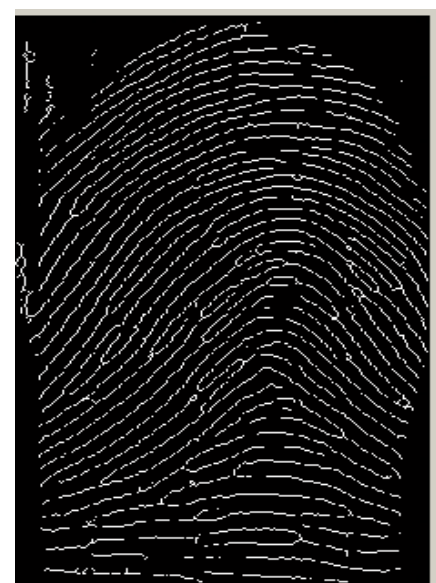

(A) Skeleton image without Secondary enhancement

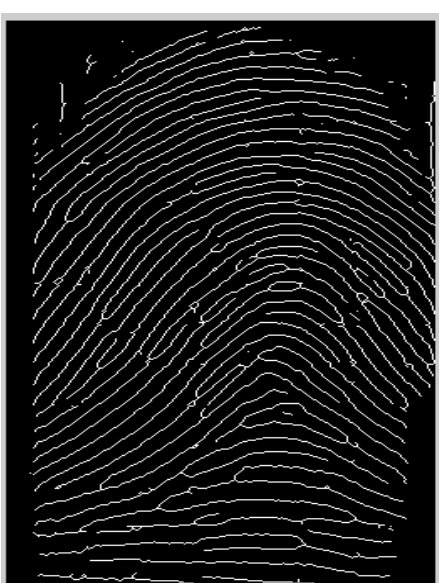

(B) Skeleton Image with Secondary enhancement

Figure 3. Skeleton image with and without secondary enhancement 
Signal \& Image Processing : An International Journal (SIPIJ) Vol.3, No.2, April 2012

\subsection{Remove bridge and Spur}

The width of the fingerprint skeleton is expected to be one pixel. However, this is not always true. There are still some erroneous pixel locations, where the skeleton has a two-pixel width. Parker (1991) has defined the erroneous pixel as the one with more than two 4-connected neighbors. The erroneous pixels are identified with their connection number two $(\mathrm{CN}=2)$ instead of $\mathrm{CN}>2$. And their present the fork regions where the bifurcations will be identified. Some of the outcomes when erroneous pixels are present while extracting minutiae are as follow:

a) Destroy the integrity of spurious bridges and spurs,

b) Interchange the type of minutiae character, and

c) Miss the detection of true minutiae.

Therefore, before minutiae extraction, the validation algorithm will eliminate the erroneous pixels while preserving the skeleton connectivity at the fork regions. For these purpose, the morphological operations on clean, bridge and spur are used.

\subsection{Minutiae extraction}

In order to extract minutiae, the proposed algorithm has used the connection number $(\mathrm{CN})$ which was defined by Rutovitz (1966). The equation of the connection number $(\mathrm{CN})$ for a pixel $\mathrm{P}$ is:

$$
C N=\frac{1}{2} \sum_{i=1}^{8}\left|P_{i}-P_{i+1}\right|
$$

Here $\mathrm{Pi}$ is the neighborhood of $\mathrm{P}$ with binary value 0 or 1 and $\mathrm{P}_{9}=\mathrm{P}_{1}$.

\subsection{Purifying minutiae}

The possible occurrence of the false minutiae has been explained by different authors ( Maio and Maltoni, 1997; Jain,Halici,Hayashi, Lee, Tsutsui,1999; Ratha,Chen,Jain, 1996 ). The minutiae extracted in the above section may include many spurious minutiae and may degrade the accuracy of the matching system, if they are considered as genuine minutiae. The procedure for the identification for the false minutiae and the removal of the same is given below by assuming a threshold distance $\mathrm{D}$ as the average interridge width representing the average distance between the two parellel neighbour ridges.

a) In the fingerprint pattern, the bifurcation and termination are considered in the same ridge if the distance between the two is less than the threshold distance D and removed the bifurcation and termination.

b) By identifying the two bifurcations( if any) on the same ridge with a inter distance less than the threshold distance, the two bifurcations will be removed

c) If a false minutiae due to any two terminations are identified with the following conditions: 
Signal \& Image Processing : An International Journal (SIPIJ) Vol.3, No.2, April 2012

(i) Two terminations are within the distance D and their directions are coincident with a small variation in angle and

(ii) no other termination is located between the two terminations, then the identified false minutiae, by the broken ridge due to two terminations, are removed.

d) The two terminations located in a short ridge with a distance less than $\mathrm{D}$ are identified and removed.

\subsection{Fingerprint matching}

The fingerprint matching is done according to He, Tian, Luo and Zhang (2003) approach. Their work is originated from Jain , Lin, Bolle (1997). The ridge information and minutiae are used for comparing two fingerprint image. While matching, if two minutiae of same type located under variable bonding box then it is consider as in the same location. A variable bonding box is more robust for non linear deformation instead of fixed size bonding box (Jain, Lin, Bolle, 1997). The matching score represent the possible similarity between the two fingerprints. According to the forensic guidelines, 12 minutiae matched is required for minimum score of successful matching for fingerprint identification (Jea, T., Govindaraju, V. 2005).

\section{EXPERIMENTAL RESULTS}

The proposed method is implementation in MATLAB 7.1 and tested using FVC2004 database. This database contains hundreds of fingerprint images, taken from different fingerprint scanners. The experimental results are analyzed by considering the following parameters and the results are depicted in table 1:

True Minutiae $\left(\mathbf{M}_{\mathbf{T}}\right)$ : Minutiae mark by a human expert.

Extracted Minutiae $\left(\mathbf{M}_{\mathbf{E}}\right)$ : Minutiae after post-processing.

False Minutiae $\left(\mathbf{M}_{\mathbf{F}}\right)$ : Minutiae extracted by the algorithm that do not coincide with $\mathrm{M}_{\mathrm{T}}$.

Dropped Minutiae $\left(\mathbf{M}_{\mathbf{D}}\right)$ : Minutiae marked by a human expert that are not extracted by the algorithm.

Type-exchange Minutiae $\left(\mathbf{M}_{\mathrm{TE}}\right)$ : Minutiae extracted by the algorithm that coincide with $\mathrm{M}_{\mathrm{T}}$ except the minutiae type 
Signal \& Image Processing : An International Journal (SIPIJ) Vol.3, No.2, April 2012

Table1: The performance evaluation of the proposed method using different fingerprint images

\begin{tabular}{|c|c|c|c|c|c|}
\hline Name(*.tif) & $\begin{array}{c}\text { True } \\
\text { Minutiae } \\
\left(\mathbf{M}_{\mathbf{T}}\right)\end{array}$ & $\begin{array}{c}\text { Extracted } \\
\text { Minutiae } \\
\left(\mathbf{M}_{\mathbf{E}}\right)\end{array}$ & $\begin{array}{c}\text { False Minutiae } \\
\left(\mathbf{M}_{\mathbf{F}}\right)\end{array}$ & $\begin{array}{c}\text { Dropped } \\
\text { Minutiae } \\
\left(\mathbf{M}_{\mathbf{D}}\right)\end{array}$ & $\begin{array}{c}\text { Type- } \\
\text { exchange } \\
\text { Minutiae } \\
\left(\mathbf{M}_{\mathbf{T E}}\right)\end{array}$ \\
\hline 1_5 & 31 & 49 & 24 & 13 & 2 \\
\hline 1_6 & 29 & 44 & 21 & 11 & 3 \\
\hline 2_1 & 22 & 42 & 16 & 7 & 1 \\
\hline $2 \_4$ & 26 & 39 & 19 & 9 & 1 \\
\hline $2 \_7$ & 24 & 62 & 46 & 5 & 4 \\
\hline $3 \_1$ & 36 & 48 & 17 & 12 & 2 \\
\hline 3_4 & 25 & 47 & 16 & 5 & 1 \\
\hline $8 \_8$ & 48 & 66 & 37 & 16 & 5 \\
\hline
\end{tabular}

The Average error rate of false minutiae is $M_{F} / M_{E}$, dropped minutiae is $M_{D} / M_{T}$ and type exchange minutiae is $\mathrm{M}_{\mathrm{TE}} / \mathrm{M}_{\mathrm{E}}$. The total error rate is the sum of them ( Zhao, Tang, 2007) and Total error rate of the proposed algorithm is $1.184 \%$. The execution time is 1.9 seconds. Figure 4. shows the different fingerprint patterns, after processing through the different stages of the proposed method.

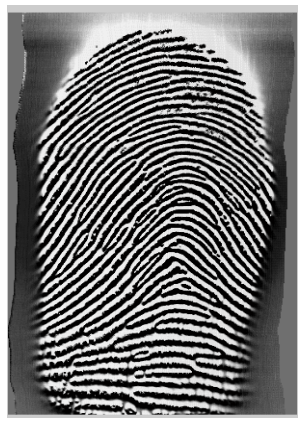

(a)

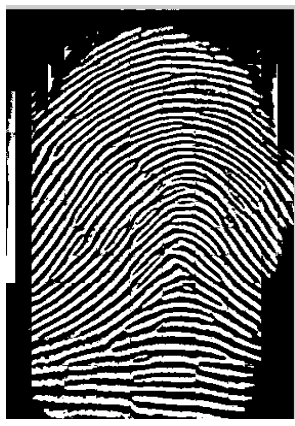

(d)

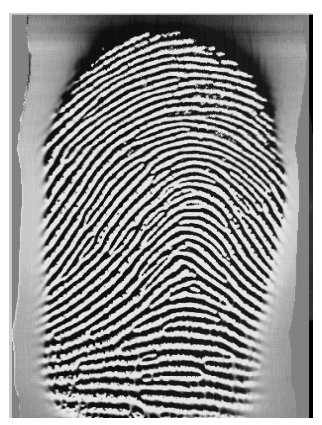

(b)

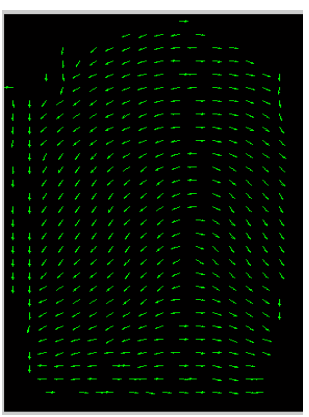

(e)

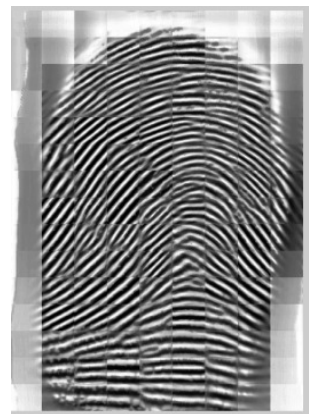

(c)

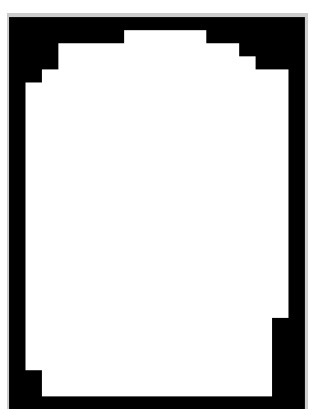

(f)

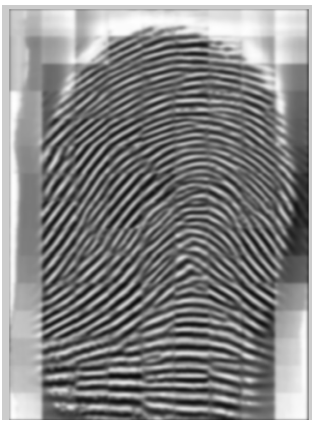

(d)

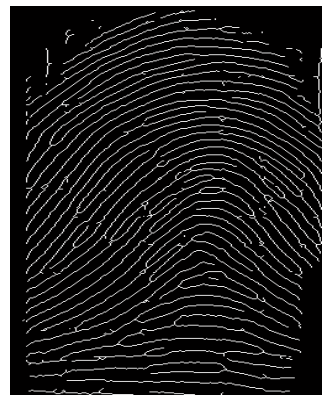

(g) 
Signal \& Image Processing : An International Journal (SIPIJ) Vol.3, No.2, April 2012

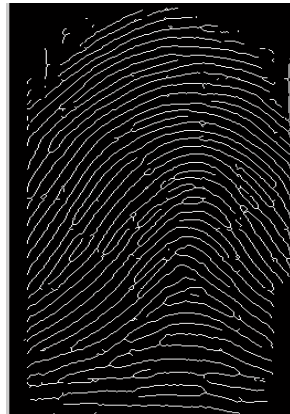

(h)

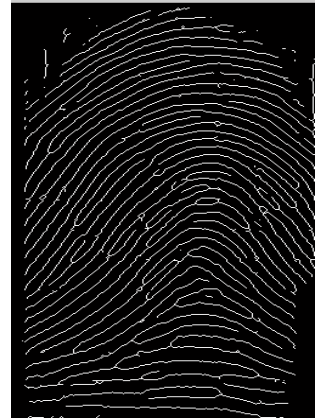

(i)

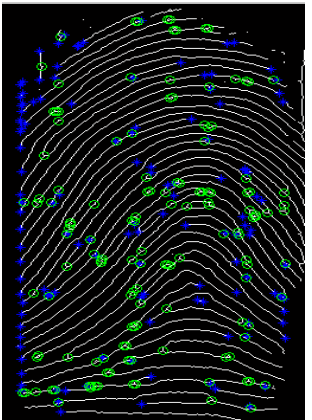

(j)

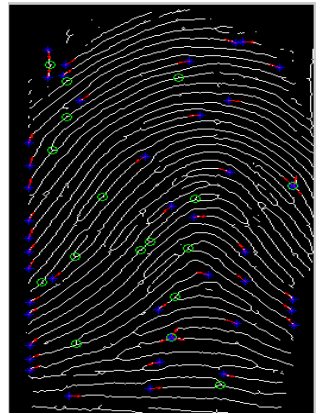

(k)

Figure 4. The different steps of implementation as shown in the above algorithm.

a) Input fingerprint image, b) Normalization image, c) Primary Enhancement, d) Secondary enhancement, e) ridge orientation, f) region of interest, g) thinning, h) remove bridge, i) remove spur, j) minutiae extraction, f) purifying minutiae.

\section{CONCLUSION}

We have proposed an effective algorithm for the fingerprint minutiae extraction with a secondary enhancement in the preprocessing stage, using a Gaussian filter after Fast Fourier transformation. The Gaussian filter makes the ridge smoothness and reduces the "hairy" structure in the ridge at the thinning process. This gives a less effort in the post processing stage while spurious minutiae are being removed. The total error rate of the proposed algorithm is within experimental limits and the proposed algorithm shows that the accuracy of fingerprint matching is reasonably improved.

\section{REFERENCES}

[1] Chen, Z. and Kuo, C.H., 1992. "A topology-based matching algorithm for fingerprint authentication", IEEE, 2, pp. 84.

[2] Douglas Hung, D.C., 1993. "Enhancement and feature purification of fingerprint images", Pattern Recognition, 26(11), pp. 1661-1671.

[3] Gabor, D., 1946. “Theory of communication”, Journal of IEE, 92, pp. 429-457.

[4] He, Y., Tian, J., Luo, X. and Zhang, T., 2003. "Image enhancement and minutiae matching in fingerprint verification”, Pattern Recognition, 24, pp. 1349-1360.

[5] Hong, L., Jain, A.K., Pankanti, S. and Bolle, R., 1996. "Fingerprint enhancement", IEEE, 5, pp. 202207.

[6] Hong, L., Wan, Y. and Jain, A. K., 1998. "Fingerprint image enhancement: algorithm and performance evaluation", IEEE Transactions on Pattern Analysis and Machine Intelligence, 20(8), pp. 777-789. 
Signal \& Image Processing : An International Journal (SIPIJ) Vol.3, No.2, April 2012

[7] Lee, H. C. and Gaensslen, R.E., 1991. “Advance in fingerprint technology”, Elsevier, New York.

[8] Mehtre, B.M., 1993. "Fingerprint image analysis for automatic identification". Machine Vision and Applications, 6, pp.124-139.

[9] Moayer, B. and Fu, K.S., 1986. "A tree system approach for fingerprint recognition", IEEE Trans. Pattern Anal. Mach. Intell. , 8(3), pp. 376-387.

[10] O`Gorman, L. and Nickerson, J.V. 1989. "An approach to fingerprint filter design”, Pattern Recognition,22(1), pp. 29-38.

[11] Parker, J.R., 1991.'Grey Level thresholding in badly illuminated images", IEEE Trans. Pattern Anal. Mach. Intell. , 13(8), pp. 813-837.

[12] Rajkumar, R. and Hemachandran, K., 2011. "A review on image enhancement of fingerprint using Directional filters", Assam University Journal of Science and Technology, 7(2), pp. 52-57.

[13] Rutovitz, D., 1966. “Pattern recognition”. J. Roy. Stat. Soc., 129, pp. 504-530.

[14] Sherlock, B.G., Monro, D.M., and Millard, K., 1994. "Fingerprint enhancement by directional Fourier filtering”, IEE Proc. Vis Image Signal Processing, 141(2), pp. 87-94.

[15] Wuzhili, 2002. "Fingerprint Recognition", Thesis, Hong Kong, Baptist University.

[16] Xiao, Q. and Raafat, H.,1991. "Fingerprint image post-processing:A combined statistical and structural approach", Pattern Recognition, 24(10), pp. 985-992.

[17] Yang, J. Liu, L., Jiang, T. and Fan, Y., 2003. "A modified Gabor filter design method for fingerprint image enhancement”, Pattern Recognition letter, 24, pp. 1805-1817.

[18] Zhao, F. and Tang, X., 2007. "Preprocessing and Postprocessing for skeleton-based fingerprint minutiae extraction”, Pattern Recognition, 40, pp. 1270-1281.

[19] Maio, D and Maltoni, D., 1997. " Direct gray-scale minutiae detection in fingerprints", IEEE Trans. Pattern Anal. And Machine Intell., 19(1), 27-40.

[20] Jain, L.C., Halici, U., Hayashi, I., Lee, S.B., Tsutsui, S.,1999. "Intelligent biometric techniques in fingerprint and face recognition", the CRC Press.

[21] Ratha, N., Chen, S., Jain, A.K., 1996. "Adaptive Flow Orientation Based Feature Extraction in Fingerprint Images", Pattern Recognition, 28, pp. 1657-1672.

[22] Jain, A., Lin, H., Bolle, R., 1997. On-line fingerprint verification. IEEE Trans. Pattern Anal. Machine Intell. 19 (4),pp. 302-313. 
Signal \& Image Processing : An International Journal (SIPIJ) Vol.3, No.2, April 2012

[23] Arpit, D. and Namboodiri, A. 2011. "Fingerprint Feature Extraction from Gray Scale Images by Ridge Tracing”, IEEE, International Joint Conference on Biometrics (IJCB), pp. 1- 8.

[24] Zhao, Q., Zhang, L., David, Z., and Luo, N. 2009, "Direct Pore Matching for Fingerprint Recognition”, Springer, ICB 2009, LNCS 5558, pp. 597-606.

[25] Jea, T., Govindaraju, V. 2005, "Minutia-based partial fingerprint recognition system", Pattern Recognition, 38, pp.1672 - 1684

\section{Authors Profile}

Raju Rajkumar received his B.Sc. Computer Science from Gulbarga University, Karnataka in 2005 and MCA degree from Visvesvaraya Technological University, Belgaum, Karnataka in 2008.Presently, he is a Research Scholar in Computer Science Department, Assam University, Silchar, Assam. His area of research interests are Biometrics, Pattern Recognition and Neural Network.

Prof. K. Hemachandran is associated with the Dept. of Computer Science, Assam University, Silchar, since 1998. He obtained his M.Sc. Degree from Sri Venkateswara University, Tirupati and M.Tech. and PhD Degree from Indian School of Mines, Dhanbad. His area of research interest are Image Processing, Soft Engineering and Distributed Computing.
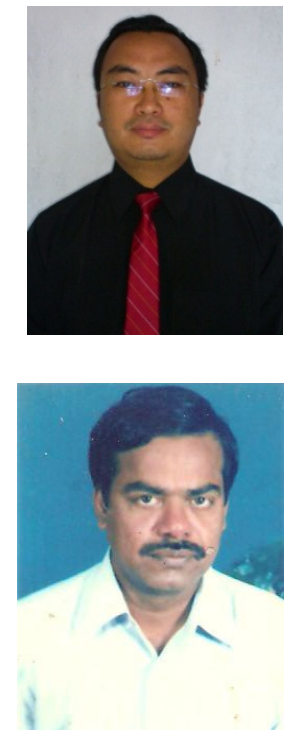